\title{
Feasibility study in the combination of non-contrast computed tomography and transcranial Doppler for large vessel occlusion detection
}

\author{
Amber Y Dorn*, Samuel G Thorpe, Kian Jalaleddini, Seth J Wilk, Robert B Hamilton \\ Novasignal Corporation, Los Angeles, CA, 90064, USA
}

\begin{abstract}
Background and purpose: Accurate detection of Large Vessel Occlusion (LVO), and subsequent assessment of candidacy for endovascular intervention, present challenging clinical obstacles to efficient stroke patient triage, especially when computed-tomography angiogram (CTA) is not readily available especially in nonprimary or comprehensive stroke centers. The Alberta Stroke Program Early CT Score (ASPECTS) is used to evaluate a patient's non-contrast CT (NCCT) exam for evidence of cerebral tissue damage and provides information about the tissue affected by stroke to determine course of treatment. Velocity curvature index (VCI) is a biomarker derived from the morphological analysis of transcranial Doppler (TCD) waveforms and has shown utility in TCD assessment of LVO. The purpose of this study was to evaluate the relationship between ASPECTS and VCI and to explore their combined diagnostic capabilities. We hypothesized that ASPECTS' assessment of LVO can be augmented with TCD imaging modality for the purpose of faster door-to-intervention workflow and triage of LVO patients to stroke centers.
\end{abstract}

Methods: NIHSS, standard of care, and TCD imaging were collected from two clinical populations; 33 LVO (16 female) and 33 In-Hospital Controls (13 female) enrolled consecutively at a regional stroke center from October 2016 through September 2017. ASPECTS and VCI were derived from NCCT and TCD exams respectively, and retrospectively evaluated in their performance against gold standard CTA.

Results: ASPECTS was not significantly correlated with VCI and predicted LVO with accuracy, sensitivity, and specificity of $82 \%, 97 \%$, and $66 \%$ while VCI performed with $91 \%, 91 \%$, and $88 \%$. Combining ASPECTS and VCI resulted in an accuracy of $91 \%$, sensitivity of $97 \%$, and specificity of $85 \%$.

Conclusions: VCI and ASPECTS' lack of correlation but independent strengths in LVO assessment indicate that they may shed light on differing mechanisms of stroke pathology.

\section{Introduction}

Acute ischemic strokes often result from a large vessel occlusion (LVO) of one of the major feeding arteries of the brain. Because of the brain's reliance upon these major vessels for perfusion, LVO strokes have high morbidity and mortality due to the difficulty of treatments in achieving recanalization [1]. As such, these LVO strokes carry generally worse prognoses, sometimes even despite intravenous thrombolysis [2]. When intravenous thrombolysis has been initiated but no recanalization or clinical improvement has been observed, endovascular treatment (EVT) is the next course of action and is only possible at comprehensive stroke centers (CSC). Recent randomized control trials evaluating the efficacy of endovascular treatment have shown the importance of timely initiation of this surgical intervention. The results of the SEER collaboration demonstrate that a shorter time from stroke onset to reperfusion indicates potential for better outcome, which declines greatly for every minute of delay [3]. The STRATIS trial showed that an increased time from EMS arrival to puncture (onset of endovascular treatment) showed a reduced likelihood of good functional recovery [4]. This important detail in successful stroke intervention was also noted in the SWIFT-PRIME trial and HERMES meta-analysis, with significant negative effects of prolonged periods from symptom onset to mechanical thrombectomy negatively impacting outcome $[5,6]$. As recanalization becomes more attainable with increased availability of endovascular intervention, the time-sensitivity observed in these trials underlines the critical importance of accurate and minimally invasive diagnostics. Now more than ever, there is a need for rapid and conclusive stroke triage and diagnosis to help facilitate rapid and effective treatment [7].

Currently, non-contrast computed tomography (NCCT), magnetic resonance imaging (MRI), CT angiography (CTA), and magnetic resonance angiography (MRA) are the standard minimally-invasive imaging protocols trusted by physicians in acute stroke assessment. When there are no limits of machine or expert availability, or patient contraindications, CTA is the preferred method of imaging. NCCT is a standard of stroke imaging and is relied upon heavily when CTA is unavailable. Once a NCCT exam has been performed, images can be analyzed to derive a patient's tissue status using the Alberta Stroke Program Early CT Score (ASPECTS). A patient's ASPECTS is based on assessment of the tissue in the MCA watershed regions such that one point is deducted for each of 10 regions that appear hypoperfused

${ }^{*}$ Correspondence to: Amber Yvonne Eddy Dorn, Novasignal Corporation, Los Angeles, CA, 90064, USA, E-mail: amber.dorn@neuralanalytics.com

Key words: transcranial doppler, TCD, ischemic stroke, large vessel occlusion, ASPECTS

Received: April 02, 2020; Accepted: April 13, 2020; Published: April 16, 2020 
and can be used to asses a patient's candidacy for surgical intervention; a threshold of 7 is used in the prediction of both functional outcome and final infarct volume in ischemic stroke [4,8-10]. In its prediction of poor outcome, an ASPECTS threshold of 7 has been shown to reflect an overall accuracy of $65.8 \%$ [11]. Though these studies have shown that ASPECTS can provide relevant information once the presence of an LVO has been established, its ability to initially indicate LVO alone has not yet been investigated.

Although CTA is the gold standard of LVO imaging, limitations include requirement of large-bore IV access, severe allergies to iodinated contrast, and an available CTA scanner [12]. The availability of CTA at all times is recommended for both primary and comprehensive stroke centers per the Society of NeuroInterventional Surgery (SNIS), and should only be substituted with MRA when absolutely necessary [12]. Although the efficacy of CTA in stroke diagnosis is unquestionable as a gold standard and strongly endorsed, its clinical utilization remains lower than desirable. In the IMS-III trial, investigators found that only about $46 \%$ of the initial study population underwent admission baseline CTA, resulting in their amendment to the original study protocol to require its use [13]. A 2015 study of these centers found that roughly $9 \%$ of all primary stroke centers with CTA availability also met the criteria of comprehensive stroke centers with additional EVT capability on-site [14].

Transcranial Doppler (TCD) ultrasound is a noninvasive ultrasonic imaging modality that directly measures Cerebral Blood Flow Velocity (CBFV) in major arteries of the head and neck which may be used to gather information about a patients' vascular status when CTA is unavailable. Morphological characteristics of the CBFV waveform are associated with various pathologies, including LVO [15-17]. TCD is currently indicated for use in acute stroke management, having demonstrated utility in detection and localization of occlusion in numerous publications [18-21]. However, the current utilization of TCD for stroke diagnosis is limited by its user-dependence in both administration and interpretation of a given exam.

Recent research has focused on developing methods to make TCD evaluation more objective, including CBFV waveform morphological analysis and development of LVO decision criteria using TCD-only derived metrics, both of which agree with CTA with high accuracy $[17,18,22]$. Thrombolysis in Brain Ischemia (TIBI) flow grades provide a systematic method of classifying pathological waveforms as observed on a TCD exam, and comparison of pre- and post-thrombolysis TIBI scores can be used to indicate status of recanalization [17,23]. This method supplements the diagnostic process; however, the subjective nature of the assignment of these grades may be prohibitive of its accuracy and consistency. The most recent of these morphological analyses presented velocity curvature index (VCI) as a clinically-useful morphological biomarker in LVO assessment [22]. VCI is derived from the mathematical principal of space curvature as applied to the CBFV waveform, providing a quantifiable way of classifying 'blunted' waveforms that have previously been determined subjectively and shown to be associated with LVO [17,22,24].

Both NCCT and TCD have objective characteristics that have been shown to contribute valuable information to the diagnosis of LVO, but little consideration has been given to the advantage of using these techniques in conjunction with one another. Evaluating the relationship between these two variables may shed light on their individual and combined diagnostic ability. Here, we sought to evaluate the relationship between NCCT and TCD exams and explore their potential utility when combined into a simple and functionally realistic decision criteria for use in acute stroke diagnosis. We hypothesized that
ASPECTS' assessment of LVO can be augmented with TCD imaging modality for the purpose of faster door-to-intervention workflow and triage of LVO patients to stroke centers.

\section{Methods}

\section{Subject data collection}

TCD data for VCI derivation and NCCT ASPECTS scores were collected from two clinical populations (In-Hospital Control and LVO groups) enrolled consecutively at Erlanger Health Systems Southeast Regional Stroke Center in Chattanooga, TN from October 2016 through September 2017. Upon admission to the emergency department (ED), all suspected stroke patients were evaluated by a vascular neurologist using the National Institutes of Health Stroke Scale (NIHSS) and received NCCT/CT Perfusion imaging to determine the type of vascular pathology before undergoing CTA and TCD exams. All TCD exam data were collected by a single expert technician who was blinded to the CTA results. CT/A/P examinations were performed using a GE Lightspeed VCT 64-section multidetector scanner (GE Healthcare, Milwaukee, WI) with a slice thickness of $0.625 \mathrm{~mm}$, and bolus injection of $70-150 \mathrm{~mL}$ of Omnipaque 350 (GE Healthcare, Milwaukee, WI) contrast material at a rate of $4.0 \mathrm{~mL} / \mathrm{s}$. CTA images were reformatted in the coronal and sagittal planes, and $10-\mathrm{mm}$. The LVO group was made up of patients with CTA-confirmed occlusion of the M1/M2 branches of the MCA and/or ICA (proximal extracranial or terminal intracranial segments); only these occlusion locations were selected because these larger cerebral arteries are most amenable to endovascular intervention. The In-Hospital Control (IHC) group were any stroke patients that were indicated as non-LVO by admission CTA. We enrolled 66 total subjects, 33 LVO (16 female), and 33 IHC (13 female), further patient information can be found in table 1. Patients were eligible for enrollment if a complete TCD exam was acquired within 4 hours of CTA, and were excluded from enrollment if any of the following applied: 1) Head CT findings were consistent with acute primary intracranial hemorrhage; 2) Patient was hemodynamically unstable and required pharmacological support for hypotension; 3) Patient underwent partial or full craniotomy; 4) Additional intracranial pathologies present, including tumor or hydrocephalus; 5) Insufficient time was anticipated for scan as specified in study protocol; 6) Significant hemodynamic pharmacological agent present (cocaine, methamphetamine, etc.); 7) Subject under arrest for felony. Data collection and analysis of exams were approved by the University of Tennessee School of Medicine Institutional Review Board (ID: 16097). In examining differences between groups, we define statistical significance as $p<0.05$.

\section{VCI Derivation from TCD CBFV waveforms}

The mathematical curvature for each TCD beat waveform was computed from the exams by a program written in Python according to the methods described in Thorpe et al. [22]. This computation was

Table 1. Average and standard deviation of each group's assessment information

\begin{tabular}{|l|c|c|c|c|c|}
\hline & \multicolumn{3}{|c|}{ In-Hospital Control } & \multicolumn{3}{|c|}{ Large-Vessel Occlusion } & \\
\hline Variable & Mean & Std Dev & Mean & Std Dev & P \\
\hline Age (years) & 56.4 & 16.3 & 66.8 & 15.7 & $\mathrm{P}<0.001$ \\
\hline ASPECTS & 9.84 & 0.57 & 5.85 & 3.11 & $\mathrm{p}<0.001$ \\
\hline VCI & 4.95 & 1.24 & 2.66 & 0.88 & $\mathrm{P}<0.001$ \\
\hline NIHSS & 1.97 & 2.09 & 16.82 & 6.71 & $\mathrm{P}<0.001$ \\
\hline Time to TCD (minutes) & 43 & 44 & 33 & 20 & $\mathrm{P}=0.26$ \\
\hline
\end{tabular}


performed by an expert researcher who was blinded to the results of each patient's NCCT and CTP results. Multiple MCA velocities across different depths between 45 and $60 \mathrm{~mm}$ were recorded trans temporally, and the VCI values were extracted by choosing the minimum of the bilateral curvature values associated with the maximal mean CBFV value from a subject's exam [22]. VCI is the summed curvature possessed by a single CBFV pulse waveform, thus assigning a lower numerical value to a waveform that may appear 'blunted' to an interpreter (Figure 1). This value provides a diagnostic biomarker that is representative of more subjectively-classified morphology parameters at its useful threshold of 3.61, chosen to maximize Youden's J statistic described in Thorpe et al. [22].

\section{Individual metric performance analysis}

We correlated ASPECTS and VCI in each group using Pearson's correlation coefficient to understand what, if any, relationship exists between these two stroke assessment techniques. In addition, differences between groups in ASPECTS and VCI were tested for significance using the Mann-Whitney U Test. We evaluated the strength of agreement between these two measures by finding the Cohen Kappa Statistic based upon the incidence of agreement in designation. From these results, the sensitivity (SEN), specificity (SPE) and accuracy (ACC) values for each metric were computed to serve as a comparison for the performance of their combination. Additionally, we generated receiver operating characteristic (ROC) curves for the selection of the optimal thresholds of VCI and ASPECTS. Distribution analyses, correlation, and ROC curves were computed using standard python libraries; SciPy Stats version 1.0 and Scikit-learn version 0.19.1.

\section{Decision criteria formation}

We used a threshold of 3.61 for VCI and 7 for ASPECTS, as have been previously published $[8,22]$. The optimal VCI threshold of 3.61 was derived from the same population data used in this analysis [22]. An ROC curve was generated to determine the optimal ASPECTS threshold for use on this specific dataset, which was in line with published clinical studies [8]. For subjects whose VCI or ASPECTS fell below either of these thresholds, they were appended to the predicted LVO group. Any subjects who did not fall below these thresholds were designated as IHC. The decisions were presented as a binary set, and the sensitivity, specificity, and accuracy values were generated based on comparison to the CTA ground-truth labels.

IHC

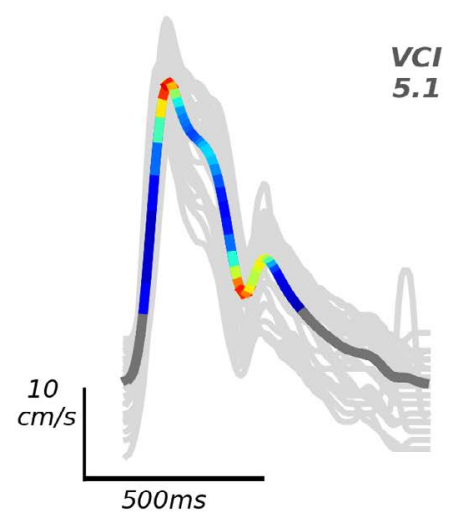

Figure 1. Example of IHC and LVO averaged CBFV waveforms, and their corresponding VCI values. LVO is associated with a lower VCI value, as described in this figure from Thorpe et al. 2018, used with permission.

\section{Results}

\section{Subject information and demographics}

Of the possible subjects with adequate initial screenings, $50 \mathrm{LVO}$ and 38 IHC were initially enrolled. In the LVO group, three were discontinued (either expressed desire to discontinue or were transferred or died). An additional $14 \mathrm{LVO}$ and five IHC subjects were excluded due to the presence of disqualifying criteria not known upon initial enrollment. Within the LVO group, there were 20 subjects with M1 occlusions, three with M2 occlusions, and eight ICA occlusions. One additional subject had tandem occlusions in the M1 and ICA of the same hemisphere, and one other subject had bilateral ICA occlusions in addition to an M2 occlusion.

For subject group demographics and attributes, see table 1 in the previous section. Significant differences in ASPECTS, VCI, and NIHSS between the IHC and LVO groups were observed upon comparison. The ranges of these metrics in each group, shown in figure 2.

\section{ASPECTS and VCI are not Correlated}

In terms of Pearson's correlation, we found no statistically significant correlation between these metrics in either experimental group; in LVO, $\mathrm{r}=0.249(\mathrm{P}=0.14)$; in IHC, $\mathrm{r}=0.127(\mathrm{P}=0.48)$ (figure 3a). ASPECTS and VCI agreed in the correct label of 29 of 33 IHC, and in 22 of 33 LVO subjects. We evaluated the Cohen Kappa Statistic for the agreement of VCI and ASPECTS and found it to be 0.46 .

\section{Performance of ASPECTS and VCI in LVO Classification}

Applied to our subject population, a threshold of 7 (in which ASPECTS $>7$ suggests non-LVO stroke) was used. In this study, the performance of ASPECTS when used at this threshold has ACC, SEN, SPE of $82 \%, 66 \%$, and $97 \%$, respectively. ASPECTS alone predicted 1 IHC subject as LVO, and $11 \mathrm{LVO}$ subjects as control (figure 3b). The ROC area-under-the-curve (AUC) value for ASPECTS was 0.88 .

Across all subjects, the mean VCI value was $3.80 \pm 1.56$. Using the same threshold value as the initial validation of VCI (3.61), VCI has ACC, SEN, SPE of $91 \%, 91 \%$, and $88 \%$, respectively (Figure 3c). The ROC AUC value for VCI was 0.94 .

\section{Combination of ASPECTS and VCI in LVO Classification}

When we combined these two metrics and their thresholds into a conditionally two-part decision process, we saw an increase from either metric in sensitivity without major detriment to specificity, with our criteria correctly identifying 32 of 33 LVO strokes (Figure 3d). This is the most clinically imperative feature to improve, as the identification of LVO can determine the method of intervention to follow. From these results, it is evident that the inclusion of VCI has the capacity to strengthen the diagnostic capacity of non-invasive LVO imaging protocols.

Using this diagnostic decision criteria in prediction of LVO and IHC labels, it performed with ACC, SEN, and SPE values of 91\%, 97\%, and $85 \%$, respectively.

\section{Discussion}

The uncorrelated relationship between ASPECTS and VCI was interesting, as these two metrics are useful in LVO assessment. Their independent accuracies and their abilities to be used together, suggest that ASPECTS and VCI may be sensitive to differing aspects of LVO pathology; their combined use may allow for a more comprehensive diagnostic assessment of these patients. 

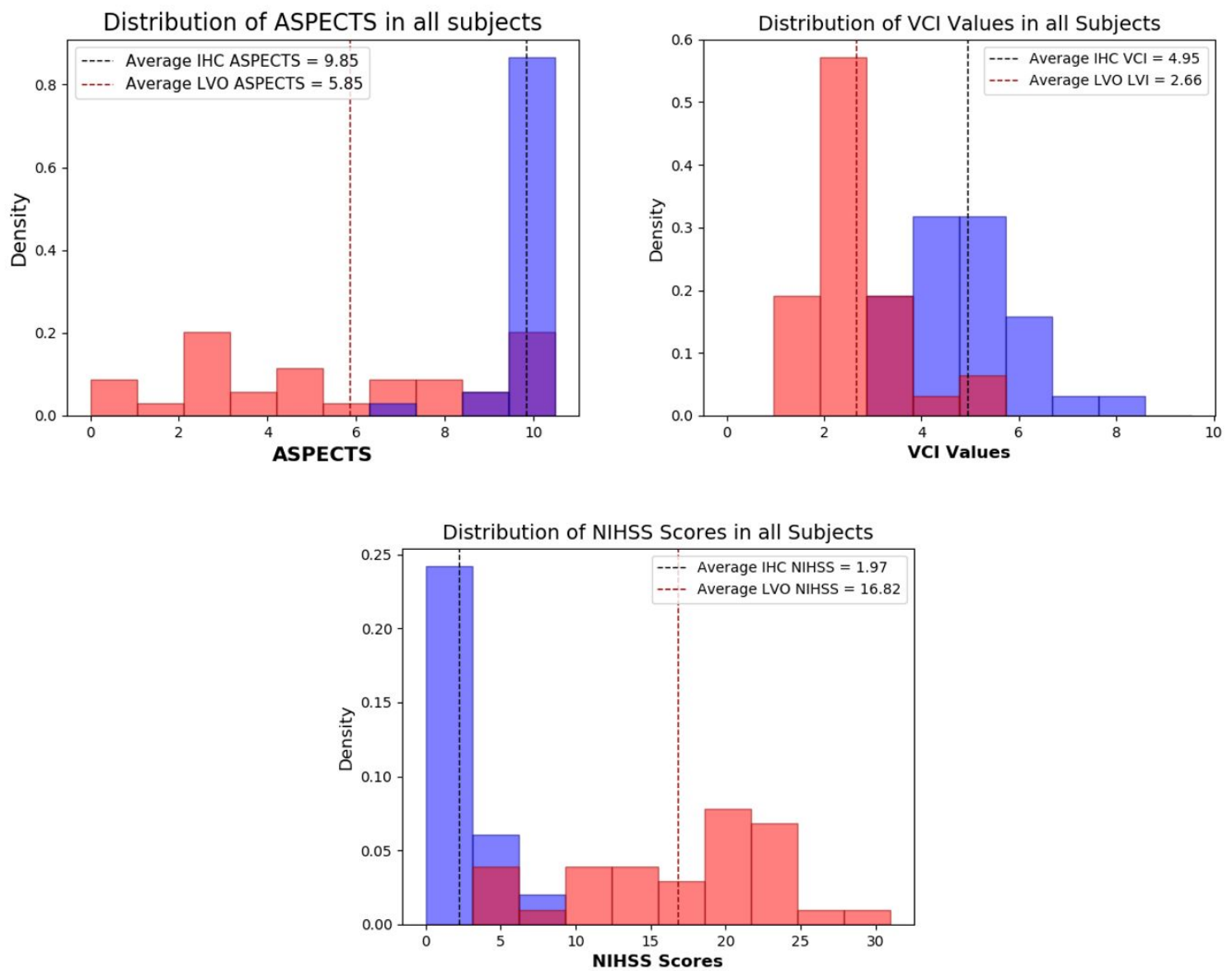

Figure 2. Distributions of (a) Alberta Stroke Program Early CT Scores (ASPECTS), (b) Velocity curvature index (VCI) values, and (c) National Institutes of Health Stroke Scale (NIHSS) Scores in both large vessel occlusion (LVO) and in-hospital control (IHC) groups

a)

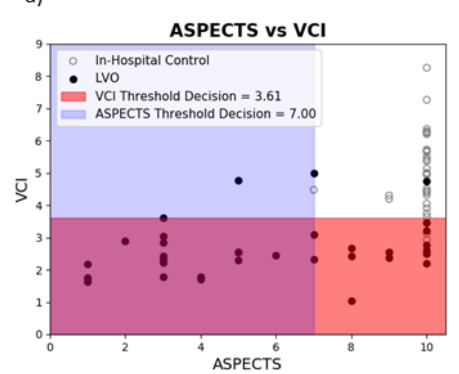

c)

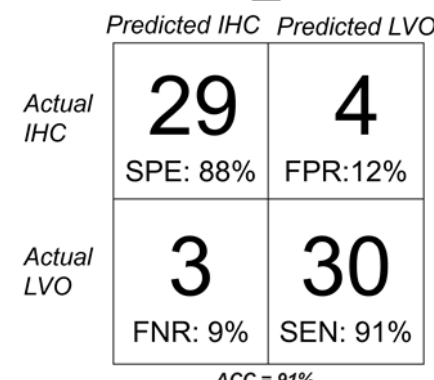

b) ASPECTS

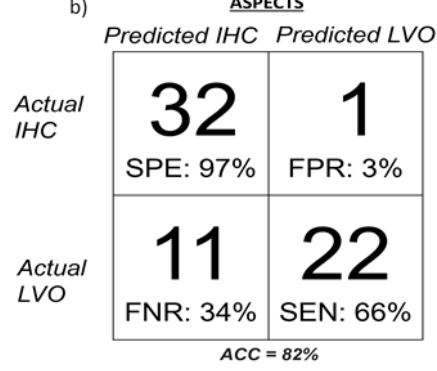

d) Decision Criteria

Predicted IHC Predicted LVO

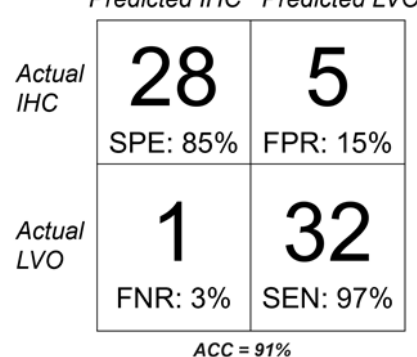

Figure 3. a) Scatter plot of CTA-confirmed IHC or LVO subjects against the decision thresholds of both ASPECTS and VCI; false-negative rate (FNR) and false-positive rates (FPR) also included. b) Independent performance of ASPECTS in LVO prediction as compared to CTA. c) Performance of VCI alone in LVO prediction against CTA. d) Performance of ASPECTS and VCI together in the separation of IHC versus LVO stroke patients. 


\section{ASPECTS provides value in noninvasive LVO diagnosis}

ASPECTS has unquestionable value in the assessment of suspected LVO patients and their candidacy for intervention, especially given its minimal impact on door-to-intervention workflow. However, its low sensitivity in this analysis could mean missed opportunity for optimal treatment of LVO patients if vascular imaging is not available. The moderately accurate performance of ASPECTS using this threshold for LVO prediction is markedly higher than previously published performance of ASPECTS for other aspects of assessment at this threshold, such as outcome prediction or infarct volume estimation $[25,26]$. A short time from symptom onset or good collateral flow are potential confounds of ASPECTS, and future studies should examine the relationship between ASPECTS and these variables.

The increase in sensitivity observed when ASPECTS and VCI are combined could bring clarity to cases which have conflicting or uncertain results from preliminary assessment tools. For example, one of the CTA confirmed LVO subjects that ASPECTS missed had presented to the ED four hours after symptom onset after transfer from an outside facility. The patient had received an unknown dosage of tPA in transfer, and CTA confirmed occlusion of the right distal M1 segment of the MCA with distal reconstitution. Upon admission, their NIHSS was 12 and their ASPECTS was 10. Presence and flow in collateral arteries were also noted. The subject was administered additional tPA, and mechanical thrombectomy achieved recanalization of TICI 2B. At 24-hour follow up, their NIHSS score increased to 15, although this could also have been due to interrater variability. VCI of 2.6 was later derived from their acute TCD exam which his below the adopted threshold of 3.61 .

\section{TCD has a long history in LVO assessment}

TCD has been present in stroke assessment since its adaptation for intracranial vessels; however, its user-dependence in both the administration and interpretation of exams has prevented it from evolving beyond its current perceived utility. Although progress has been made in the device's size and bulk, these new technologies still require expert insonation or supervision [27,28]. Although automated TCD manipulation and analysis is a relatively new development, there is potential for TCD to be simplified and made less operator independent such that if successful it could be more easily used in a prehospital or centers without CTA. To mitigate the problem of subjective interpretations of exams, signal processing techniques such as VCI are being developed and provide promising and reliable options [22]. Factors such as prior infarcts may be missed by TCD but would likely present in an NIHSS evaluation or NCCT exam.

Although VCI is an independently strong biomarker of LVO, its potential utility lies in the information it can add to the standard diagnostic battery of non-invasive stroke assessments; here the VCI metric alone did not suggest LVO in three CTA confirmed subjects, however, referencing NIHSS and ASPECTS only missed one. As an example, one presented to the ED with NIHSS of 24 and ASPECTS of 7, both of which suggested LVO diagnosis. CTA confirmed right proximal M1 MCA occlusion. CTA also noted presence and patent bilateral posterior communicating arteries. This subject received IV-tPA and mechanical thrombectomy. After 5 passes, recanalization of TICI 2B was achieved, and their 24-hour follow-up NIHSS score was 8 . VCI was found to be 4.98 .

\section{Combining these noninvasive techniques shows promise for centers lacking CTA}

Although CTA is required to image intracranial vasculature and will remain the gold standard for centers with access, the results of this analysis indicate that ASPECTS and VCI may indicate differing but complimentary facets of stroke pathology. When utilized together, these metrics could provide a more comprehensive and sensitive method of LVO diagnosis in the absence of CTA. Based on the improved performance of the combination of these metrics, VCI may be able to contribute contextual information regarding a patient's vascular status that allows ASPECTS to provide more clarity.

The single LVO subject on which VCI and ASPECTS agreed on predicting as a false negative (IHC; 4.73 and 10, respectively) had an acute NIHSS score of 13. CTA identified a right terminal ICA occlusion and displayed very good collateral flow. Radiology notes from this subject's NCCT noted evidence of subacute or chronic frontal lobe infarction. The presence of good collateral flow, if strong enough, could act as a confound for both VCI and ASPECTS due to the absence of perfusion differences that are typically evident on both TCD and NCCT exams.

\section{Study limitations}

The performance of this decision criteria may be limited by the small sample size, and abnormally distributed sample populations. The largely-separate distributions of NIHSS and ASPECTS in the IHC and LVO groups may undermine the true differential ability of our decision criteria; for this reason, the analysis should next be applied to a larger, more variable, study population. In a sample population where more overlap between experimental group occurs for each evaluated metric, the performance of each threshold may be better understood to maximize their utility in this criterion.

Although even CBFV morphological analyses are influenced by the angle of insonation, methods including VCI seek to mitigate this limitation by using the maximal velocity, which is assumed to be the most-true value, for its computation. Additionally, the novelty of VCI limits the experimental possibilities without a well-established threshold to refer to, so subsequent evaluations of it are difficult to draw conclusions from. As VCI is used in a larger variety of diagnostic circumstances, its optimal threshold for LVO detection can be tested and verified.

\section{Acknowledgements}

The authors are very grateful for the advice and expertise of Dr. Corey M. Thibeault, Ph.D. for his contributions to the concept design and data analysis.

\section{Funding Information}

The data collected and analyzed in this study was part of a feasibility study designed and funded by Neural Analytics, Inc.

\section{References}

1. Hill MD, Barber PA, Demchuk AM, Newcommon NJ, Cole-Haskayne A, et al. (2002) Acute Intravenous-Intra-Arterial Revascularization Therapy for Severe Ischemic Stroke. Stroke 33: 279-82. [Crossref]

2. Newell DW, Grady SM, Eskridge JM, Winn RH (1990) Distribution of Angiographic Vasospasm after Subarachnoid Hemorrhage: Implications for Diagnosis by Transcranial Doppler Ultrasonography. Neurosurgery 27: 574-577. [Crossref]

3. Campbell BC, Hill MD, Rubiera M, Menon BK, Demchuk A, et al. (2016) Safety and Efficacy of Solitaire Stent Thrombectomy Individual Patient Data Meta-Analysis of Randomized Trials. Stroke 47: 798-806. [Crossref] 
4. Mueller-Kronast NH, Zaidat OO, Froehler MT, Jahan R, Aziz-Sultan MA, et al. Systematic Evaluation of Patients Treated With Neurothrombectomy Devices for Acute Ischemic Stroke Primary Results of the STRATIS Registry. Stroke 48: 27602768. [Crossref]

5. Saver JL, Goyal M, van der Lugt A, Menon BK, Majoie CB, et al. (2016) Time to Treatment With Endovascular Thrombectomy and Outcomes From Ischemic Stroke: A Meta-analysis. JAMA 316: 1279. [Crossref]

6. Saver JL, Goyal M, Bonafe A, Diener H-C, Levy I, et al. (2015) Stent-Retriever Thrombectomy after Intravenous t-PA vs. t-PA Alone in Stroke. $N$ Engl $J$ Med 24:2285-2295. [Crossref]

7. Saver JL (2006) Time Is Brain-Quantified. Stroke 37: 263-266. [Crossref]

8. Coutts SB, Lev MH, Eliasziw M, Roccatagliata L, Hill MD, et al. (2004) ASPECTS on CTA Source Images Versus Unenhanced CT Added Value in Predicting Final Infarct Extent and Clinical Outcome. Stroke 35: 2472-6. [Crossref]

9. Demeestere J, Garcia-Esperon C, Garcia-Bermejo P, Ombelet F, McElduff P, et al. (2017) Evaluation of hyperacute infarct volume using ASPECTS and brain CT perfusion core volume. Neurology 88: 2248-2253. [Crossref]

10. Naylor J, Churilov L, Chen Z, Koome M, Rane N, et al. (2017) Reliability, Reproducibility and Prognostic Accuracy of the Alberta Stroke Program Early CT Score on CT Perfusion and Non-Contrast CT in Hyperacute Stroke. Cerebrovasc Dis 44: 195-202. [Crossref]

11. González RG, Lev MH, Goldmacher G V., Smith WS, Payabvash S, et al. (2012) Improved Outcome Prediction Using CT Angiography in Addition to Standard Ischemic Stroke Assessment: Results from the STOPStroke Study. Kiechl S, ed. PLoS One 7: e30352. [Crossref]

12. McTaggart RA, Ansari SA, Goyal M, Abruzzo TA, Albani B, et al. (2015) Initial hospital management of patients with emergent large vessel occlusion (ELVO): report of the standards and guidelines committee of the Society of NeuroInterventional Surgery. J Neurointerv Surg 9: 316-323. [Crossref]

13. Broderick JP, Palesch YY, Demchuk AM, Yeatts SD, Khatri P, et al. (2013) Endovascular Therapy after Intravenous t-PA versus t-PA Alone for Stroke. $N$ Engl $J$ Med 368: 893-903. [Crossref]

14. Schieb LJ, Casper ML, George MG (2015) Mapping Primary and Comprehensive Stroke Centers by Certification Organization. Circ Cardiovasc Qual Outcomes 8: S193-4. [Crossref]

15. Christou I, Felberg RA, Demchuk AM, Grotta JC, Burgin WS, et al. (2001) A Broad Diagnostic Battery for Bedside Transcranial Doppler to Detect Flow Changes With Internal Carotid Artery Stenosis or Occlusion. J Neuroimaging 11: 236-242. [Crossref]
16. Demchuk AM, Christou I, Wein TH, Felberg RA, Malkoff M, et al. (2000) Accuracy and criteria for localizing arterial occlusion with transcranial Doppler. J Neuroimaging 10: 1-12. [Crossref]

17. Demchuk AM, Burgin WS, Christou I, Felberg RA, Barber PA, et al. (2001) Thrombolysis in Brain Ischemia (TIBI) Transcranial Doppler Flow Grades Predict Clinical Severity, Early Recovery, and Mortality in Patients Treated With Intravenous Tissue Plasminogen Activator. Stroke 32: 89-93. [Crossref]

18. Thorpe SG, Thibeault CM, Canac N, Wilk SJ, Devlin T, et al. (2018) Decision Criteria for Large Vessel Occlusion Using Transcranial Doppler Waveform Morphology. Front Neurol 9: 847. [Crossref]

19. Demchuk AM, Christou I, Wein TH, Felberg RA, Malkoff M, et al. (2000) Specific transcranial Doppler flow findings related to the presence and site of arterial occlusion. Stroke 31: 140-146. [Crossref]

20. Tsivgoulis G, Sharma VK, Lao AY, Malkoff MD, Alexandrov AV (2007) Validation of Transcranial Doppler With Computed Tomography Angiography in Acute Cerebral Ischemia. Stroke 38: 1245-9. [Crossref]

21. Burgin WS, Malkoff M, Felberg RA, Demchuk AM, Christou I, et al. (2000) Transcranial Doppler Ultrasound Criteria for Recanalization After Thrombolysis for Middle Cerebral Artery Stroke. Stroke 31: 1128-32. [Crossref]

22. Thorpe SG, Thibeault CM, Wilk SJ, O’Brien M, Canac N, et al. (2018) Velocity Curvature Index: a Novel Diagnostic Biomarker for Large Vessel Occlusion. Transl Stroke Res 10: 475-484. [Crossref]

23. Rubiera M, Ribo M, Pagola J, Coscojuela P, Rodriguez-Luna D, et al. (2011) Bridging Intravenous-Intra-Arterial Rescue Strategy Increases Recanalization and the Likelihood of a Good Outcome in Nonresponder Intravenous Tissue Plasminogen ActivatorTreated Patients A Case-Control Study. Stroke 42: 993-997. [Crossref]

24. Thorpe SG, Thibeault CM, Canac N, Jalaleddini K, Dorn A, et al. (2020) Toward automated classification of pathological transcranial Doppler waveform morphology via spectral clustering. PLoS One 15: e228642. [Crossref]

25. van Seeters T, Biessels GJ, Kappelle LJ, van der Schaaf IC, Dankbaar JW, et al. (2015) The Prognostic Value of CT Angiography and CT Perfusion in Acute Ischemic Stroke. Cerebrovasc Dis 40: 258-269. [Crossref]

26. Lin K, Lee SA, Zink WE (2011) What ASPECTS Value Best Predicts the 100-mL Threshold on Diffusion Weighted Imaging? Study of 150 Patients with Middle Cerebral Artery Stroke. J Neuroimaging 21: 229-231. [Crossref]

27. TCD Monitoring - Delica Transcranial Ultrasound Doppler - Products - SMT medical $\mathrm{GmbH}$ \& Co. Available from: https://www.smt-medical.com/en/products/delicatranscranial-ultrasound-doppler/tcd-monitoring.html. Accessed September 30, 2019.

28. Pietrangelo SJ, Lee HS, Sodini CG (2018) A Wearable Transcranial Doppler Ultrasound Phased Array System. Acta Neurochir Suppl 126: 111-114. [Crossref]

Copyright: (C2020 Dorn AY. This is an open-access article distributed under the terms of the Creative Commons Attribution License, which permits unrestricted use, distribution, and reproduction in any medium, provided the original author and source are credited. 